\title{
To AND or To OR: How Shall the Fusion Center Rule in Energy-Constrained Cognitive Radio Networks?
}

\author{
Sina Maleki ${ }^{\dagger}$, Geert Leus ${ }^{\star}$, Symeon Chatzinotas ${ }^{\dagger}$ and Björn Ottersten ${ }^{\dagger}$ \\ ${ }^{\dagger}$ Interdisciplinary Centre for Security, Trust and Reliability (SnT), \\ University of Luxembourg, \\ Email: \{sina.maleki, symeon.chatzinotas, bjorn.ottersten\}@uni.lu \\ *Faculty of Electrical Engineering, Mathematics and Computer Science, \\ Delft University of Technology, \\ Email: g.j.t.leus@tudelft.nl
}

\begin{abstract}
Distributed spectrum sensing enhances the detection reliability of a cognitive radio network. However, this comes at the price of a higher energy consumption. To solve this problem, a combined censoring and sleeping scheme is considered where the cognitive radios switch off their sensing module with a specific sleeping rate in each sensing period. The awake cognitive radios send their local decisions to the fusion center only if it is deemed to be informative. The fusion center either employs the OR or the AND rule to make the final decision about the presence or absence of the primary user. This paper investigates which rule performs better in terms of energy efficiency under various conditions. The underlying sensing parameters are derived by minimizing the maximum average energy consumption per sensor subject to a constraint on the probabilities of false alarm and detection. This way, it can be ensured that the spectrum opportunities are utilized efficiently while the primary users are not interfered with. A case study based on IEEE 802.15.4/ZigBee is considered for performance evaluation. It is shown that significant energy savings can be obtained by employing combined censoring and sleeping.
\end{abstract}

\section{INTRODUCTION}

Distributed spectrum sensing improves the detection performance of a cognitive radio network [1], [2]. The cognitive radios sense the spectrum in periodic sensing slots and send processed data to the fusion center (FC) based on their observations. The FC is then responsible to make the final decision about the presence or absence of the primary user. The received processed data at the FC is either soft data such as likelihood ratio test (LRT) results or binary hard decisions. A comprehensive study of several soft and hard fusion schemes is provided in [3]. Due to its energy efficiency as well as implementation simplicity, a binary hard fusion scheme is considered in this paper. We focus on two famous decision rules known as the OR and AND rules. The OR rule decides for the presence of the primary user, if at least one cognitive radio reports as such, while the AND rule dictates the absence of the primary user if at least one cognitive radio reports the absence.

Here, we consider cognitive radio networks which consist of low-power sensor networks with limited batteries. This way, designing energy-efficient algorithms to perform different tasks in a cognitive radio system is a critical issue. Note that we assume the FC has unlimited energy access, and thus we only consider optimizing energy consumption at the cognitive radios. We focus on designing an energy-efficient algorithm for distributed spectrum sensing in a network, where the FC employs either the OR or the AND rule. A combined censoring and sleeping scheme is considered. The cognitive radios randomly switch off their sensing module with a specific sleeping rate. While awake, each cognitive radio employs a censoring policy to send their decisions to the FC. By applying censoring, we make sure that only those decisions which are deemed to be informative, are transmitted to the FC. Corresponding to the censoring policy, there is a censoring region which is defined by a lower threshold $\lambda_{1}$, and an upper one $\lambda_{2}$. In this paper, we determine the lower and upper thresholds as well as the sleeping rate by minimizing the maximum average energy consumption per sensor subject to a lower bound on the probability of detection and an upper bound on the probability of false alarm. Therefore, the cognitive radio network avoids making harmful interference to the primary user activities while efficiently exploiting the spectrum opportunities. We show that the optimal average energy consumption per sensor is achieved by $\lambda_{1}=0$ for the OR rule, and $\lambda_{2} \rightarrow \infty$ for the AND rule. Exploiting this, we simplify the underlying problems and show that the optimization problem for each rule can be solved by a bounded line search.

In [4]-[6] censoring is considered for distributed detection in sensor networks. The underlying parameters including the upper and lower thresholds are obtained in two ways. The probability of miss detection is minimized subject to a constraint on the probability of false alarm and a limited network energy consumption in a Neyman-Pearson (NP) problem formulation, while the detection error probability is minimized subject to the network energy consumption constraint in a Bayesian problem formulation. The paper [7] considers a combination of censoring and sleeping in sensor networks from an information-theoretic viewpoint. The underlying parameters are derived by maximizing the mutual information between the state of the signal occupancy and the decision state of the FC.

Censoring for distributed spectrum sensing is considered in [8]-[12]. The communication overhead of the cognitive radio network is reduced by employing a censoring policy 
in [8] for an OR rule based distributed spectrum sensing scenario. In [9], the maximum average energy consumption per sensor is minimized subject to a specific detection performance constraint in order to design sensing parameters for a censored truncated sequential sensing scheme. The sensors sequentially collect observation samples until they can reach a decision about the presence or absence of the primary user before reaching a specific truncation point. Censoring for collaborative spectrum sensing is considered in [10] for a scenario where cognitive radios employ cyclostationary detection as their sensing technique. A combined censoring and sleeping scenario similar to the one in this paper, is discussed in [11]. The total network energy consumption is minimized subject to a specific detection performance constraint to determine the optimal censoring and sleeping rates. This way, it is shown that the network energy consumption reduces significantly. Note that in low-power sensor networks, individual energy consumption of sensors is a more critical factor. That is why in this paper, we consider minimizing the maximum average energy consumption per sensor instead of minimizing the total network energy consumption. Further, in [11], only the OR rule is considered, while in this paper, we apply this to the AND rule as well. A joint sensing and decision node selection scheme is considered in [12]. The network energy consumption is minimized subject to a detection performance constraint defined as in [11], in order to determine the sensing nodes from a pool of cognitive radios and subsequently the decision nodes from the selected sensing nodes. The decision nodes are the nodes which send their result to the FC. Finally, optimization of the network throughput for energy-constrained cognitive radios is considered in [13] in order to determine the optimal hard fusion strategies for distributed spectrum sensing. However, no energy-efficient algorithm such as censoring or sleeping is considered in [13].

The remainder of the paper is organized as follows. The system model as well as combined censoring and sleeping is introduced in Section II. We shall formulate and analyze the underlying optimization problems for the OR and AND rules in Section III, followed by numerical results in Section IV. We shall draw our conclusions in Section V.

\section{SySTEM MODEL}

A network of $M$ cognitive radios (CRs) is considered where cognitive radios gain spectrum access if the primary user is not active within a certain band. Cognitive radios sense the frequency band in periodic sensing slots, and solve a binary hypothesis testing problem by collecting $N$ samples in order to determine the status of the band regarding the presence or absence of the primary user. The underlying hypotheses are defined as $\mathcal{H}_{0}$ and $\mathcal{H}_{1}$ indicating the absence and presence of the primary user, respectively. The local results are then sent to the FC where the final decision is made as shown in Fig. 1. Denoting $s_{i}$ to be the primary user signal at time $i$ with zeromean and variance $\sigma_{s}^{2}, w_{i j}$ to be the additive white Gaussian noise with zero-mean and variance $\sigma_{w}^{2}, h_{j}$ to be the channel gain between the primary user and cognitive radio (assumed to be constant during each sensing period), and $r_{i j}$ to be the $i$-th received sample at the $j$-th cognitive radio, the binary hypothesis testing problem is formulated as follows

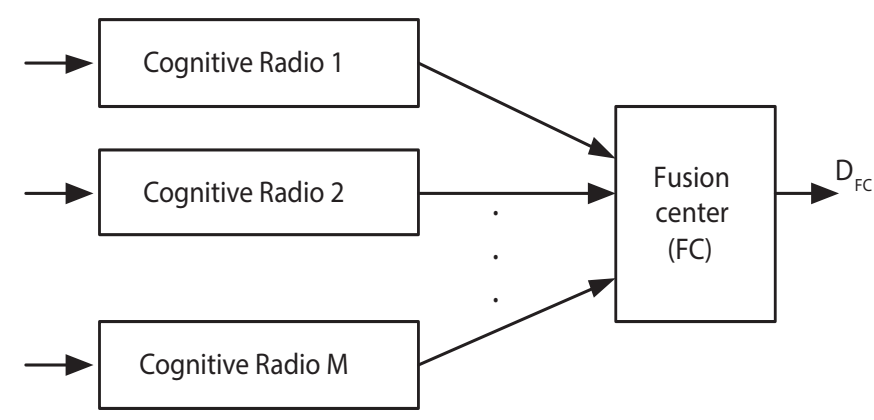

Fig. 1: Distributed spectrum sensing configuration

$$
\begin{aligned}
& \mathcal{H}_{0}: r_{i j}=w_{i j}, \\
& \mathcal{H}_{1}: r_{i j}=h_{j} s_{i}+w_{i j} .
\end{aligned}
$$

Each cognitive radio employs an energy detector which calculates the accumulated energy of $N$ samples, as follows

$$
\mathcal{E}_{j}=\sum_{i=1}^{N} \frac{\left|r_{i j}\right|^{2}}{\sigma_{w}^{2}}
$$

A censoring policy is then applied to solve (1), which is defined by a lower threshold $\lambda_{1}$ and an upper threshold $\lambda_{2}$ in order to determine the informative region of $\mathcal{E}_{j}$. The calculated $\mathcal{E}_{j}$ in (2) is only considered to be informative, if $\mathcal{E}_{j} \geq \lambda_{2}$ or $\mathcal{E}_{j} \leq \lambda_{1}$. This way, the decision rule at the $j$-th cognitive radio is given by

$$
\left\{\begin{array}{lr}
\text { send } 1 \text {, declaring } \mathcal{H}_{1} & \text { if } \mathcal{E}_{j} \geq \lambda_{2} \\
\text { no decision } & \text { if } \lambda_{1}<\mathcal{E}_{j}<\lambda_{2} \\
\text { send } 0, \text { declaring } \mathcal{H}_{0} & \text { if } \mathcal{E}_{j} \leq \lambda_{1}
\end{array}\right.
$$

Considering the model in $(1), \mathcal{E}_{j}$ follows a chi-squared distribution with $N$ degrees of freedom under $\mathcal{H}_{0}$ and $\mathcal{H}_{1}$. This way, the local probabilities of false alarm $P_{f, j}$ and detection $P_{d, j}$ are derived as

$$
\begin{gathered}
P_{f, j}=\frac{\Gamma\left(N, \frac{\lambda_{2}}{2}\right)}{\Gamma(N)}:=P_{f}, \\
P_{d, j}=\frac{\Gamma\left(N, \frac{\lambda_{2}}{2\left(1+\gamma_{j}\right)}\right)}{\Gamma(N)} .
\end{gathered}
$$

where $\gamma_{j}=\frac{\left|h_{j}\right|^{2} \sigma_{s}^{2}}{\sigma_{w}^{2}}$ is the signal to noise ratio (SNR) at the $j$-th cognitive radio. We further define the censoring rate of a cognitive radio as $\rho_{j}=\operatorname{Pr}\left(\lambda_{1}<\mathcal{E}_{j}<\lambda_{2}\right)$, where $\operatorname{Pr}$ denotes probability. Here, we assume that the a priori probabilities of primary user presence or absence represented by $\pi_{0}=\operatorname{Pr}\left(\mathcal{H}_{0}\right)$ and $\pi_{1}=\operatorname{Pr}\left(\mathcal{H}_{1}\right)$, are known. This way, denoting $\delta_{0, j}$ and $\delta_{1, j}$ as the censoring rates under $\mathcal{H}_{0}$ and $\mathcal{H}_{1}$, respectively, we obtain $\rho_{j}=\pi_{0} \delta_{0, j}+\pi_{1} \delta_{1, j}$, where $\delta_{0, j}$ and $\delta_{1, j}$ are obtained by

$$
\delta_{0, j}=\frac{\Gamma\left(N, \frac{\lambda_{1}}{2}\right)}{\Gamma(N)}-\frac{\Gamma\left(N, \frac{\lambda_{2}}{2}\right)}{\Gamma(N)}:=\delta_{0},
$$




$$
\delta_{1, j}=\frac{\Gamma\left(N, \frac{\lambda_{1}}{2\left(1+\gamma_{j}\right)}\right)}{\Gamma(N)}-\frac{\Gamma\left(N, \frac{\lambda_{2}}{2\left(1+\gamma_{j}\right)}\right)}{\Gamma(N)} .
$$

On top of censoring, in each sensing period, each cognitive radio switches off its sensing module with probability $\mu$, the sleeping rate. Therefore, on top of the transmission energy savings which are achieved by employing censoring, the energy which is consumed during the sensing time shall also be reduced. Denoting $C_{s, j}$ to be the sensing energy per sample and $C_{t, j}$ to be the transmission energy per bit at the $j$-th cognitive radio, the average energy consumption at the $j$-th cognitive radio becomes

$$
C_{j}=(1-\mu)\left(N C_{s, j}+C_{t, j}\left(1-\rho_{j}\right)\right) .
$$

We assume that $\mu \neq 0$ and $\rho_{j} \neq 0$.

The FC decides for $\mathcal{H}_{0}$ and $\mathcal{H}_{1}$ by employing either an OR rule or an AND rule. These two rules are the simplest hard fusion rules to implement in a distributed detection scenario. The OR rule based FC decides for $\mathcal{H}_{1}$ if at least one cognitive radio sends a 1 to the $\mathrm{FC}$ while in case of the AND rule, the FC votes for $\mathcal{H}_{0}$ if at least one cognitive radio reports a 0 . In the following section, the global probabilities of false alarm and detection for both rules are derived, and the underlying problem formulations are discussed.

\section{ANALYSis AND PRoBlem Formulation}

Our goal is to minimize the average energy consumption per sensor. However, since minimizing the average energy consumption per sensor for each individual cognitive radio becomes NP-hard, we consider minimizing the maximum average energy consumption per sensor. We further need to take the cognitive radio requirements regarding the interference limitations and throughput into account. As mentioned earlier, the cognitive radio should avoid harmful interference to the primary user. A constraint on the global probability of detection is then enforced by this requirement. Further, to increase the cognitive network throughput, correct detection of a spectrum hole is critical and thus the probability of false alarm shall be designed as low as possible. We define an upper bound denoted by $\alpha$ for the global probability of false alarm, $Q_{\mathrm{F}}$, and a lower bound denoted by $\beta$ for the global probability of detection, $Q_{\mathrm{D}}$. Our goal is then to design the system parameters including $\lambda_{1}, \lambda_{2}$ and $\mu$ by minimizing the maximum average energy consumption per sensor subject to a constraint on the global probabilities of false alarm and detection, as follows

$$
\begin{aligned}
& \min _{\mu, \lambda_{1}, \lambda_{2}} \max _{j} C_{j} \\
& \text { s.t. } Q_{\mathrm{F}} \leq \alpha, Q_{\mathrm{D}} \geq \beta .
\end{aligned}
$$

In this section, first, we derive $Q_{\mathrm{F}}$ and $Q_{D}$, followed by an analysis of (9) for the OR rule in Section III-A, and then we do the same for the AND rule in Section III-B.

\section{A. OR rule}

In this sub-section, the FC employs the OR rule in order to make the final decision. Denoting $D_{\mathrm{FC}}$ to be the decision made at the FC, the OR rule means $D_{\mathrm{FC}}=1$ if at least one cognitive radio sends a 1 , else $D_{\mathrm{FC}}=0$. This way, the global probability of false alarm $Q_{\mathrm{F}, \mathrm{OR}}$ for the $\mathrm{OR}$ rule is obtained by

$$
\begin{aligned}
Q_{\mathrm{F}, \mathrm{OR}} & =\operatorname{Pr}\left(D_{\mathrm{FC}}=1 \mid \mathcal{H}_{0}\right) \\
& =1-\operatorname{Pr}\left(D_{\mathrm{FC}}=0 \mid \mathcal{H}_{0}\right) \\
& =1-\prod_{j=1}^{M}\left[1-(1-\mu) P_{f}\right] \\
& =1-\left[1-(1-\mu) P_{f}\right]^{M},
\end{aligned}
$$

where $P_{f, j}$ is given by (4). This can be easily explained by the OR rule based global probability of false alarm when considering $(1-\mu) P_{f}$ to be the local probability of false alarm including the sleeping policy. Note that the false alarm and detection rates are independent from censoring, because if a sensor does not transmit a result to the FC, the FC assumes that the decision of this sensor is zero.

The global probability of detection $Q_{\mathrm{D}, \mathrm{OR}}$ for the OR rule can be derived in a similar way, and results in

$$
\begin{aligned}
Q_{\mathrm{D}, \mathrm{OR}} & =\operatorname{Pr}\left(D_{\mathrm{FC}}=1 \mid \mathcal{H}_{1}\right) \\
& =1-\operatorname{Pr}\left(D_{\mathrm{FC}}=0 \mid \mathcal{H}_{1}\right) \\
& =1-\prod_{j=1}^{M}\left[1-(1-\mu) P_{d, j}\right]
\end{aligned}
$$

where $P_{d, j}$ is given by (5). This also can be explained by the OR rule based global probability of detection when considering $(1-\mu) P_{d, j}$ to be the local probability of detection including the sleeping policy.

To analyze (9) for the OR rule, it is more convenient to rewrite it in the following format

$$
\begin{aligned}
& \min _{\mu, \lambda_{1}, \lambda_{2}} \max _{j}(1-\mu)\left[N C_{s, j}+C_{t, j}\left(1-\rho_{j}\right)\right] \\
& \text { s.t. } 1-\left[1-(1-\mu) P_{f}\right]^{M} \leq \alpha \\
& \quad 1-\prod_{j=1}^{M}\left[1-(1-\mu) P_{d, j}\right] \geq \beta .
\end{aligned}
$$

Since for the OR rule, the FC only decides on the presence of the primary user by receiving $1 \mathrm{~s}$, sending $0 \mathrm{~s}$ is not optimal in terms of energy efficiency. Therefore, $\lambda_{1}=0$ is the optimal solution to (12). Using this result, we can relax one of the arguments of the problem. When $\lambda_{1}=0$, we obtain

$$
\begin{aligned}
& 1-\delta_{0}=P_{f}, \\
& 1-\delta_{1, j}=P_{d, j} .
\end{aligned}
$$

Hence, (12) is given by

$$
\begin{aligned}
& \min _{\mu, \lambda_{2}} \max _{j}(1-\mu)\left[N C_{s, j}+C_{t, j}\left(\pi_{0} P_{f}+\pi_{1} P_{d, j}\right)\right] \\
& \text { s.t. } 1-\left[1-(1-\mu) P_{f}\right]^{M} \leq \alpha \\
& \quad Q_{\mathrm{D}, \mathrm{OR}} \geq \beta .
\end{aligned}
$$


After some simplifications, we can show that our optimization problem reduces to the following line search problem

$$
\begin{gathered}
\max _{j} \min _{\mu}(1-\mu)\left[N C_{s, j}+C_{t, j}\left(\pi_{0} F\left(G^{-1}(\mu, \beta)\right)\right.\right. \\
\left.\left.+\pi_{1} F\left(G^{-1}(\mu, \beta) /\left(2\left(1+\gamma_{j}\right)\right)\right)\right)\right] .
\end{gathered}
$$

where $F(x)=\frac{\Gamma(N, x)}{\Gamma(N)}, G\left(\mu, \lambda_{2}\right)=Q_{\mathrm{D}, \mathrm{OR}}$, and $\mu$ should satisfy $F\left(G^{-1}(\mu, \beta)\right) \leq \alpha^{\prime} /(1-\mu)$, where $\alpha^{\prime}=1-(1-\alpha)^{1 / M}$ and $G^{-1}$ is the inverse function defined over the second argument in $G\left(\mu, \lambda_{1}\right)$. Denoting $\mu^{*}$ as the solution of (15), the optimal probability of false alarm is obtained by $P_{f}^{*}=F\left(G^{-1}\left(\mu^{*}, \beta\right)\right)$. Deriving the optimal $\lambda_{2}$ is then straightforward.

\section{B. AND Rule}

Here, we analyze the performance of combined sleeping and censoring for the AND rule. According to the AND rule, $D_{\mathrm{FC}}=0$, if at least one cognitive radio reports a zero, else $D_{\mathrm{FC}}=1$. Note that for the AND rule, if the FC receives no decision from the $j$-th cognitive user, it automatically considers this decision to be 1 . This way, the global probabilities of false alarm and detection are obtained as follows

$$
\begin{aligned}
Q_{\mathrm{F}, \mathrm{AND}} & =\operatorname{Pr}\left(D_{\mathrm{FC}}=1 \mid \mathcal{H}_{0}\right) \\
& =\prod_{j=1}^{M}\left[\mu+(1-\mu)\left(\delta_{0}+P_{f}\right)\right] \\
& =\left[\mu+(1-\mu)\left(\delta_{0}+P_{f}\right)\right]^{M} \\
& =\left[1-(1-\mu)\left(1-\delta_{0}-P_{f}\right)\right]^{M} . \\
Q_{\mathrm{D}, \mathrm{AND}} & =\operatorname{Pr}\left(D_{\mathrm{FC}}=1 \mid \mathcal{H}_{1}\right) \\
= & \prod_{j=1}^{M}\left[\mu+(1-\mu)\left(\delta_{1, j}+P_{d, j}\right)\right] \\
= & \prod_{j=1}^{M}\left[1-(1-\mu)\left(1-\delta_{1, j}-P_{d, j}\right)\right] .
\end{aligned}
$$

These derivations can be easily explained by the AND rule based global probabilities of false alarm and detection when considering $1-\left[(1-\mu)\left(1-\delta_{0}-P_{f}\right)\right]$ and $1-\left[(1-\mu)\left(1-\delta_{1, j}-\right.\right.$ $\left.P_{d, j}\right)$ to be the local probabilities of false alarm and detection including the sleeping and censoring policies, respectively. Note that for the AND rule, the FC considers any result except 0 as 1 . Therefore, from the FC viewpoint, a false alarm (or detection) at the $j$-th cognitive radio occurs if the received result is not 0 . That is why for the AND rule, the censoring rate plays a role in the global probabilities of false alarm and detection which is not the case for the OR rule.

We define our problem in order to find the underlying arguments $\left(\lambda_{1}, \lambda_{2}, \mu\right)$, so as to minimize the maximum average energy consumption per sensor subject to a constraint on the probabilities of false alarm and detection. As in the previous scenario, the constraints on the probabilities of false alarm and detection are defined by an upper bound $\alpha$ and a lower bound $\beta$, respectively. This way, the problem is written as follows

$$
\begin{aligned}
& \min _{\mu, \lambda_{1}, \lambda_{2}} \max _{j} C_{j} \\
& \text { s.t. } Q_{\mathrm{F}, \mathrm{AND}} \leq \alpha, Q_{\mathrm{D}, \mathrm{AND}} \geq \beta .
\end{aligned}
$$

Since the FC decides for $\mathcal{H}_{0}$ only by receiving zeros, the optimal solution of (18) is attained by $\lambda_{2} \rightarrow \infty$. This way, the global probabilities of false alarm and detection reduce to

$$
\begin{gathered}
Q_{\mathrm{F}, \mathrm{AND}}=\left[1-(1-\mu)\left(1-\delta_{0}\right)\right]^{M}, \\
Q_{\mathrm{D}, \mathrm{AND}}=\prod_{j=1}^{M}\left[1-(1-\mu)\left(1-\delta_{1, j}\right)\right] .
\end{gathered}
$$

Inserting (19) and (20) in (18) and relaxing $\lambda_{2}$ using the fact that $\lambda_{2} \rightarrow \infty$ is optimal, we obtain

$$
\begin{aligned}
& \min _{\mu, \lambda_{1}} \max _{j}(1-\mu)\left(N C_{s, j}+C_{t, j}\left(1-\rho_{j}\right)\right) \\
& \text { s.t. } {\left[1-(1-\mu)\left(1-\delta_{0}\right)\right]^{M} \leq \alpha } \\
& \prod_{j=1}^{M}\left[1-(1-\mu)\left(1-\delta_{1, j}\right)\right] \geq \beta,
\end{aligned}
$$

where $\rho_{j}=\pi_{0} \delta_{0}+\pi_{1} \delta_{1, j}$. After some derivations, we obtain the following line search problem in order to determine the optimal $\mu$ and consequently $\delta_{0}$ and $\lambda_{1}$

$$
\begin{gathered}
\min _{\mu} \max _{j}(1-\mu)\left[N C_{s, j}+C_{t, j}\left(1-\pi_{0}\left(1-\frac{1-\alpha^{1 / M}}{1-\mu}\right)\right.\right. \\
\left.\left.-\pi_{1} F_{j, \mathrm{AND}}\left(1-\frac{1-\alpha^{1 / M}}{1-\mu}\right)\right)\right]
\end{gathered}
$$

where $F_{j, \mathrm{AND}}\left(\delta_{0}\right)=\delta_{1, j}\left(\delta_{0}\right)=F\left(F^{-1}\left(\delta_{0}\right) /\left(1+\gamma_{j}\right)\right)$. In search for the optimal $\mu$, we should note that $\mu \leq \alpha^{1 / M}$ which comes from the fact that $1-\frac{1-\alpha^{1 / M}}{1-\mu} \geq 0$ and also $G(\mu, 1-$ $\left.\frac{1-\alpha^{1 / M}}{1-\mu}\right) \geq \beta$. Denoting $\mu^{*}$ as the optimal solution of (22), the optimal $\delta_{0}$ is obtained by $\delta_{0}^{*}=1-\frac{1-\alpha^{1 / M}}{1-\mu^{*}}$. Determining $\lambda_{1}$ is then straightforward.

\section{NUMERICAL RESUlTS}

In this section, we evaluate the performance of the proposed combined censoring and sleeping scheme for the OR and AND rules in several scenarios regarding the probability of primary user absence (presence). To determine the sensing and transmission energy of each cognitive radio, a Chipcon CC2240 transceiver based on the IEEE 802.15.4/ZigBee standard is considered [14]. We assume that all the cognitive radios experience the same SNR which is equal to $\gamma=10 \mathrm{~dB}$. The 


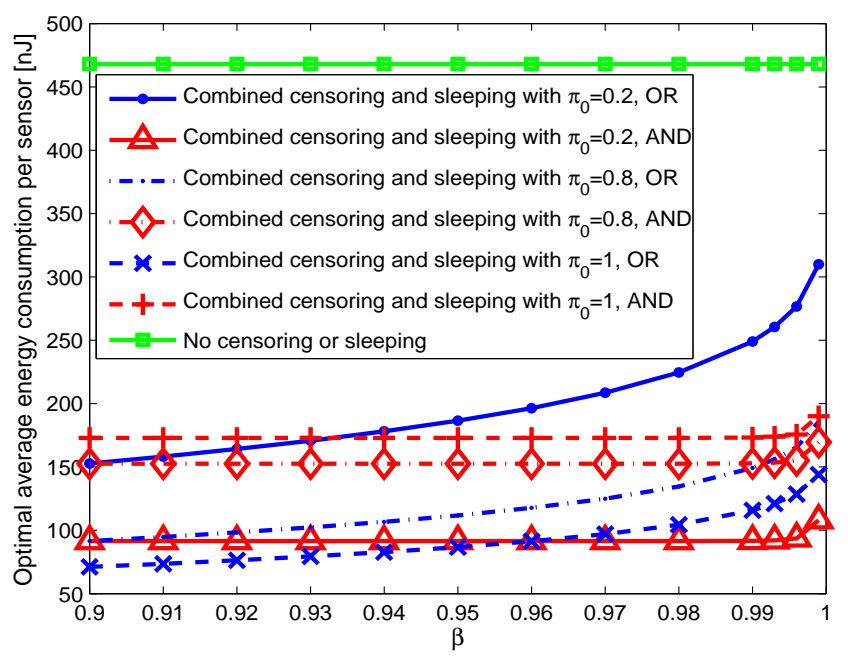

Fig. 2: Optimal average energy consumption per sensor versus $\beta$ for different setups.

number of samples is assumed to be $N=5$ which corresponds to a sensing time of $1 \mu \mathrm{s}$. Cognitive radios are uniformly distributed around a circle with radius $70 \mathrm{~m}$ and the $\mathrm{FC}$ is located at the centre. A free-space path loss model is employed to model the wireless channel between the cognitive radio and the FC and this leads to a signal attenuation which is inversely proportional to the square of the distance $d$ between the transmitter and receiver. The total sensing energy consisting of listening and processing energy for 5 samples is derived in [11] and is roughly equal to $5 C_{s}=190 \mathrm{~nJ}$. Following the same model as in [11], the transmission energy for a range of $70 \mathrm{~m}$ and transmission of one-bit decision, is approximately $C_{t}=278 \mathrm{~nJ}$. Note that the transmission energy is derived in order to satisfy a receiver sensitivity of $-90 \mathrm{dBm}$ at a SNR of $10 \mathrm{~dB}$.

Fig. 2 depicts the optimal average energy consumption per sensor versus the probability of detection constraint, $\beta$. The number of cognitive radios is $M=5$, the probability of false alarm constraint $\alpha=0.1$ and $0.9 \leq \beta \leq 0.99$. We let $\pi_{0}$ adapt to three values including $\{0.2, \overline{0} .8, \overline{1}\}$ reflecting the cases where the probability of primary user absence is low, high, and extremely high, respectively. We can see that the combined censoring and sleeping scheme delivers a high energy saving compared to the scenario where no energy-efficient scheme is considered. We further notice that the AND rule outperforms the OR rule for low values of $\pi_{0}$ reflecting the lower chance of reporting 0 and thus a higher censoring rate compared to the OR rule as shown in Fig. 3. The opposite trend is shown for the case where $\pi_{0}$ is high. For example, except for the high values of $\beta$, the OR rule outperforms the AND rule when $\pi_{0}=0.8$. For the extremely high values of $\pi_{0}$, it is shown that the OR rule always outperforms the AND rule with much higher censoring rate as shown in Fig. 3. It is also shown that the lower bound on the optimal average energy consumption per sensor for the OR rule is achieved when $\pi_{0}=1$.

The optimal average energy consumption per sensor is drawn in Fig. 4 versus the number of cognitive radios. In this

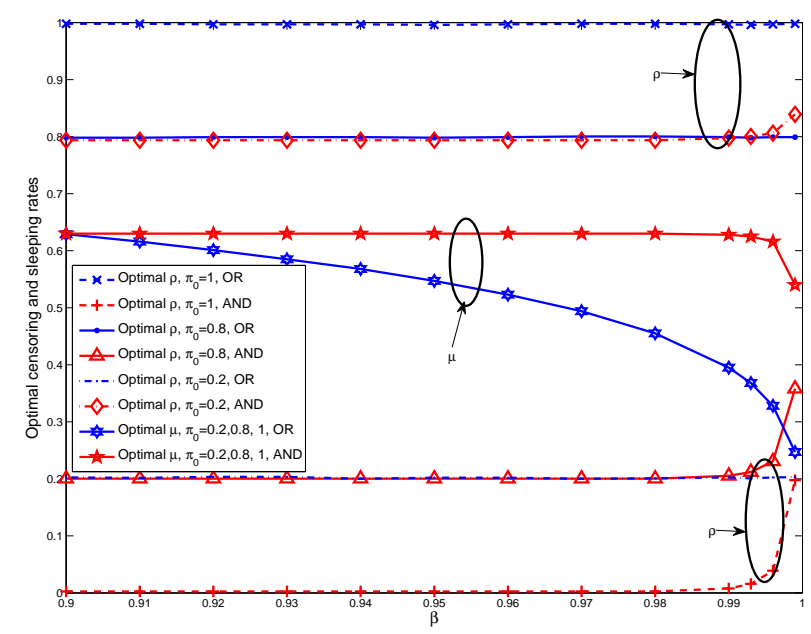

Fig. 3: Optimal censoring and sleeping rate versus $\beta$ for different setups

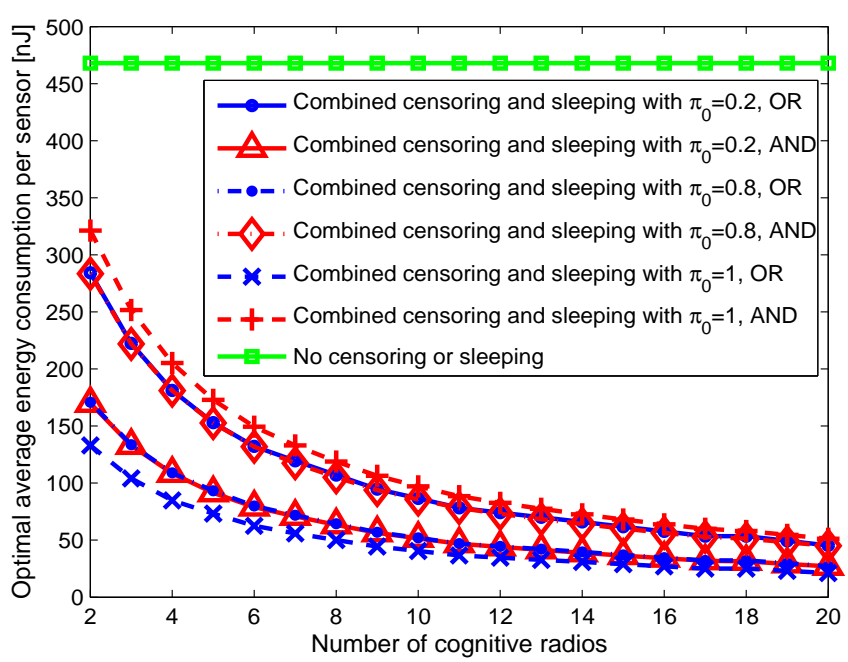

Fig. 4: Energy scaling with number of cognitive sensors for different setups.

figure, the global probabilities of false alarm and detection are assumed to be $\alpha=0.1$ and $\beta=0.9$. Again it is shown that combined censoring and sleeping is very promising in terms of energy-efficiency with respect to the scenarios where no energy-efficiency is taken into account. We can see that as the number of cognitive radios increases, the system gains a higher energy saving, inflicting a lower burden on individual cognitive radios. As in Fig. 2, the AND rule outperforms the OR rule for low values of $\pi_{0}$, while the OR rule outperforms the AND rule for high values of $\pi_{0}$. We can see again that the lower bound on the optimal average energy consumption per sensor for the OR rule is achieved when $\pi_{0}=1$. 


\section{CONCLUSiON}

In this paper, a combined censoring and sleeping scheme was discussed in order to reduce the sensing and transmission energy of the cognitive radios involved in spectrum sensing. A distributed spectrum sensing scenario was considered where the fusion center either employs the OR or AND rule. The global probabilities of false alarm and detection for each rule were derived and the underlying sensing parameters including the censoring and sleeping rates were determined by minimizing the maximum average energy consumption per sensor. It was shown that the AND rule performs very well in terms of energy efficiency in situations where the activity of the primary user is high, while the OR rule delivers a good performance in situations where the activity of the primary user is low or particularity extremely low. Therefore, we suggest designing an adaptive approach, where the fusion rule can alternate between the OR and AND rules, depending on the activity of the primary user within a specific band. We further conclude that if a cognitive radio network is well-designed in terms of energy efficiency, increasing the number of cognitive radios not only improves the detection performance but also enhances the energy efficiency of individual cognitive radios.

Note that, because of mathematical tractability, this paper only dealt with the OR and the AND rules. Asymptotic analysis of energy-efficiency considering combined censoring and sleeping for a general $K$-out-of- $M$ fusion rule is a subject of further studies.

\section{ACKNOWLEDGMENT}

This work is partially supported by NWO-STW under the VICI program (Project 10382), and the National Research Fund, Luxembourg under the project CO2SAT : Cooperative \& Cognitive Architectures for Satellite Networks.

\section{REFERENCES}

[1] E. Axell, G. Leus, E. G. Larsson, H. V. Poor, "Spectrum Sensing for Cognitive Radio : State-of-the-Art and Recent Advances," IEEE Signal Processing Magazine, vol.29, no.3, pp.101,116, May 2012

[2] S. M. Mishra, A. Sahai and R. W. Brodersen, "Cooperative Sensing among Cognitive Radios," IEEE International Conference on Communications, pp 1658-1663, June 2006.

[3] P. K. Varshney, Distributed Detection and Data Fusion, Springer, 1996.

[4] S. Appadwedula, V. V. Veeravalli and D. L. Jones, "Decentralized Detection With Censoring Sensors," IEEE Transactions on Signal Processing, pp 1362-1373, Apr 2008.

[5] S. Appadwedula, V. V. Veeravalli and D. L. Jones, "Energy-efficient detection in sensor networks," IEEE Journal on Selected Areas in Communications, pp 693-702, Apr 2005.

[6] C. Rago, P. Willett and Y. Bar-Shalom, "Censoring sensors: a lowcommunication-rate scheme for distributed detection," IEEE Transactions on Aerospace and Electronic Systems, pp 554-568, Apr 1996.

[7] K. Yamasaki and T. Ohtsuki, "Design of energy-efficient wireless sensor networks with censoring, on-off, and censoring and on-off sensors based on mutual information," IEEE Vehicular Technology Conference, pp 1312-1316, 2005.

[8] C. Sun, W. Zhang and K. B. Letaief, "Cooperative Spectrum Sensing for Cognitive Radios under Bandwidth Constraints," IEEE Wireless Communications and Networking Conference, March 2007.

[9] S. Maleki and G. Leus, "Censored Truncated Sequential Spectrum Sensing for Cognitive Radio Networks," IEEE Journal on Selected Areas in Communications, vol.31, no.3, pp.364,378, March 2013
[10] J. Lunden, V. Koivunen, A. Huttunen and H. V. Poor, "Collaborative Cyclostationary Spectrum Sensing for Cognitive Radio Systems," IEEE Transactions on Signal Processing, vol.57, no.11, pp.4182,4195, Nov. 2009

[11] S. Maleki, A. Pandharipande and G. Leus, "Energy-Efficient Distributed Spectrum Sensing for Cognitive Sensor Networks," IEEE Sensors Journal, vol.11, no.3, pp.565,573, March 2011

[12] M. Najimi, A. Ebrahimzadeh, S. M. H. Andargoli and A. Fallahi, "A Novel Sensing Nodes and Decision Node Selection Method for Energy Efficiency of Cooperative Spectrum Sensing in Cognitive Sensor Networks,” IEEE Sensors Journal, vol.13, no.5, pp.1610,1621, May 2013

[13] S. Maleki, S. P. Chepuri and G. Leus, "Optimization of hard fusion based spectrum sensing for energy-constrained cognitive radio networks", Physical Communication, Available online 20 July 2012

[14] IEEE 802.15.4 standard, Part 15.4: Wireless Medium Access Control (MAC) and Physical Layer (PHY) Specifications for Low-Rate Wireless Personal Area Networks (WPANs), 2006. 